\title{
A CASE OF APHASIA, WITH SPECIAL REFERENCE TO THE PROBLEMS OF REPETITION AND WORD-FINDING
}

\author{
BY \\ K. GOLDSTEIN and J. MARMOR \\ From the Laboratories of Neuro-Physiology, Montefiore Hospital, New York
}

(RECEIVED 3RD JUNE, 1938)

So much has been written on the subject of aphasia that the presentation of another case report requires some justification. The following case of amnesic aphasia was not only unusual in its clinical aspects, but also raised a number of important questions with regard to the phenomena of repetition and wordfinding, and to the problem of cerebral localization of language function.

\section{Case Report}

G.B., a 56-year-old white man, was admitted to the Montefiore Hospital on 25th August, 1935, with a history of a "stroke" eight months previously, followed by sudden onset of unconsciousness and paralysis of the right side of the body. $\mathrm{He}$ recovered consciousness after about one hour, seemed quite clear mentally and was able to talk coherently. Six hours later, however, he developed difficulty in speaking, although he seemed to understand what was said to him. There was bladder and rectal incontinence during the first week, but not thereafter. After one month the motor weakness, especially of the leg, improved and he was able to walk about. The speech disability, however, persisted.

The past and family history were not important except that the patient's father had died of " apoplexy" at the age of 52 .

The patient was a poorly nourished, elderly man, ambulatory, and not acutely ill. Heart sounds were regular, but of poor quality and there was a faint systolic murmur at the apex. Peripheral vessels were moderately thickened. Blood pressure was $130 / 84$.

There was a right spastic hemiparesis, with the arm in extension. The leg was less involved than the arm. Deep reflexes were increased on the right, with right ankle clonus. Abdominals and cremasterics were all present, but markedly diminished on the right. Troemner, Babinski, Rossolimo, and other confirmatory signs were present on the right. There was a mild, right-sided hemisensory syndrome, including the face, extending to the left of the mid-line, and involving all modalities. Sensory impairment was greatest in the hand. There was also distinct hyperpathia on stroking or pinching the right half of the body. The pupils reacted somewhat sluggishly to light and accommodation. The fundi were normal. There was questionable impairment of vision in the right homonymous fields. The face showed a slight right supranuclear weakness. 


\section{Mental Examination.}

1. General Behaviour.-The patient's behaviour was noteworthy. He sat quietly in a chair most of the day, took no part and showed no interest in the ward activities, and spoke not at all to his fellow-patients. However, in any situation which directly concerned him - this otherwise quiet, noncommunicative and passive individual, became animated, excited and overtalkative. He answered questions freely and co-operated to the best of his ability in all investigations. He would talk incessantly to the doctors, discussing his infirmities at great length, with much facial expression and show of emotion, laughing and crying alternately.

2. Speech.-From the history it was noted that the patient had been able to speak at least five languages fluently-German, English, Yiddish, Polish, and French. At the time of our examination, he was still able to use any of these media for expression, but favoured a combination of English, German, and Yiddish. His speech was best under conditions of emotional stress. At such times, he spoke fairly coherently and grammatically, utilizing a good vocabulary. Usually he uttered short, simple sentences which contained all types of words, although at times a subject or a verb might be misplaced. There was no paraphasia. There was a tendency to stereotypy and he frequently repeated himself. The striking thing, however, was that despite this relatively good conversational speech, he showed a complete inability to name objects or to repeat to command either letters, words or sentences. Although he might have just used a particular word or sentence in spontaneous speech, he was totally unable to repeat it to command a moment later. When asked to recite the numbers from one to ten, or the letters of the alphabet, or the days of the week, he could do so only if the first few were given to him. Then if asked to repeat one of the symbols he was unable to do so unless he recited the whole series. Thus, if after counting from one to ten, he was asked to repeat the number seven, he could do so only by counting consecutively from one to seven. Often, in his attempt to repeat a given word, he would utter other words, which invariably were related to the given word. Thus when asked to say "God" he could not do so, but would say "Himmel" (Heaven); for " children," he would say " family" ; for "hospital "-_" Montefiore" ; for " window"- " glass," etc.

He could not name even the most familiar objects although he was usually able to demonstrate or explain the use of an object that was shown to him, and sometimes uttered the correct word in the course of his explanation. When asked immediately afterward to repeat the word, however, he could never do so.

He understood many words, obeyed simple commands, was able to point to certain parts of his body as named, and could put out his tongue when asked. He also seemed to understand simple conversational sentences, although he did not comprehend more complicated ones. His understanding was always better when the spoken words fitted the particular situation which he was experiencing at the time (see Protocol I).

3. Writing.-He was able to print out his name in Latin capitals, but could 
not write any other words. He could write numbers, both single and double, to dictation, and was able to perform, in writing, simple arithmetic computations.

4. Reading.-He was able to read only his name and the heading of the New York Times. However, it seemed that this was not real reading but a recognition of familiar visual pictures, since he could not read single letters or words. He could not identify a spoken word with a written word.

He apparently recognized pictures of objects although he could not name them. Similarly, he was able to recognize objects placed in his hands (with eyes closed) and could demonstrate their use, without being able to name them.

6. General Intelligence, and State of Sensorium.-Conversations with the patient and various tests indicated that he retained a fairly good level of intellectual activity. His memory and judgement were only moderately impaired. $\mathrm{He}$ had definite appreciation of the fact that his speech was changed. Sensorium was intact, and he was oriented in all spheres. His reactions to concrete situations within his immediate environment remained fairly good.

In contrast to this, however, he was completely unable to adequately handle any situation which required a capacity for abstract conceptions (see Protocols II, III, and IV).

Laboratory Data.-Encephalography showed marked cortical atrophy, with moderate internal hydrocephalus. In one of the A.P. views there was clearly visible in the region of the left Sylvian fissure a large triangular collection of air with its base pointed outward. Gastric analysis showed free HCL of 66 , total acid of 78. Stool was positive for occult blood. G-I series indicated the presence of duodenal ulcer. Wassermann and other routine laboratory data were negative.

Course in the Hospital.-Patient complained frequently of epigastric pain, and G-I series showed evidence of a duodenal ulcer, for which he was treated medically. On 19th May, 1936, he suddenly developed signs suggestive of a ruptured duodenal ulcer. These signs subsided with 24 hours, and the abdomen became soft. However, the pains persisted, and on 25th May, 1936, laparotomy was performed. At operation, a small perforation was discovered in the first portion of the duodenum. This was sealed over, and a posterior gastro-enterostomy was performed. The patient developed signs of broncho-pneumonia on the third post operative day and expired on 29th May, 1936.

Diagnosis.-Clinical diagnosis was : thrombosis of left middle cerebral artery, ruptured duodenal ulcer and bronchopneumonia. Anatomic diagnosis was : thrombosis of left middle cerebral artery, duodenal ulcer with perforation and peritonitis, gastro-jejunostomy and bronchopneumonia.

Autopsy Observation on the Brain.-There was a large subarachnoid collection of fluid in the left temporal region, which escaped when the brain was removed. The brain weighed 1,200 grm. The vessels at the base showed moderate arteriosclerotic changes. The left hemisphere was smaller than the right, and along the line of the left Sylvian fissure there was a deep excavation (Fig. 1) with destruction of the entire superior temporal gyrus and Island of Reil, and the lowermost portions of the precentral, postcentral, supramarginal, and angular gyri. Broca's area, and the middle temporal and superior parietal convolutions were not involved. The brain was cut coronally (Figs. 2 and 3). In sections through the third ventricle, the area of softening was found to extend deeply to involve the claustrum and capsula externa, ceasing at the border of the putamen. The temporal horn of the left lateral ventricle was slightly dilated. In sections through the substantia nigra and splenium of the corpus callosum, part of the white matter of the centrum ovale was also destroyed. The area of destruction extended posteriorly to the end of the angular gyrus. There was no other lesion and no special atrophy of the brain cortex. 


\section{Summary and Discussion}

In summary, here was a man, who following a cerebral insult, showed the following picture :-Although his general intelligence was not bad, there was a marked reduction in his spontaneity. His entire personality was narrowed. He was interested only in his own needs. His contact with the outer world consisted mainly in trying to defend himself against the disturbances which arose from it. He was friendly and co-operative in all relationships which he thought might help him, and was therefore very co-operative in all investigations. $\mathrm{He}$ was able to speak five languages. His general knowledge appeared to be little disturbed. His behaviour was, in the main, adequate; the striking feature of it, was that he always reacted in a very concrete manner. In any situation, however, which required a " capacity for abstract attitude" (Gelb and Goldstein, 1924) he was completely unable to function adequately.

Perhaps the most striking symptom which the patient exhibited was his defect in speech. Within certain limitations, he was able to speak words and even sentences without paraphasia or dysarthria ; nor did he show any gross defect in understanding. There was relatively little disturbance in either the receptive or expressive aspects of his speech ; such defects in speaking and understanding as he showed, were not caused by any disturbance of the sensory or motor functions themselves, but by a deficiency in other functions, which we shall discuss.

Compared to this relative retention of his capacity for speech and understanding, it was amazing that the patient was totally lacking in the ability for repeating-either letters, words, or sentences. Inasmuch as his understanding was relatively unimpaired, this inability could not be attributed to a difficulty in hearing. Since there was no disability in motor speech, that could also be excluded as a cause. As we have mentioned, he could not repeat to command a word that he had just used spontaneously. Thus we cannot but assume that we were dealing with a disturbance of a special capacity for language repetition.

There has been a good deal of discussion about the psycho-physiological character of repetition, and of the anatomical basis for this capacity, a discussion which we cannot report here. We would refer the interested reader to the explanations of this phenomenon given by Goldstein (1927). Here we can mention only a few of the factors involved which are important for understanding the present case.

Repeating (nachsprechen) has generally been considered a very simple phenomenon. Thus Wernicke conceived of repetition of one's words as a simple overflowing excitation from the sensory to the motor part of the "speech" cortex in the form of a simple reflex arc and believed it to be based on the function of direct fibres connecting the acoustic speech centre in the temporal lobe (especially the fasciculus arcuatus). However, further investigations disclosed that this assumption does not correspond to analysis of the psychic phenomena, and that it does not agree with the anatomical findings in patients with lesions in this region. We know of cases in which these long association fibres have been destroyed, and the patient was able to repeat (Goldstein, 1915). 
Repetition is not a simple reaction of a motor apparatus to a sensory stimulation. In repeating, we must be able to perceive the complicated sound of the spoken word and to imitate it more or less voluntarily by means of complicated movements. This leads to the assumption that a simple reflex arc could not be the basis of this function, but that it is dependent upon a more complex association system. This assumption is supported by the anatomical facts. Goldstein (1915) suggested many years ago that repetition is dependent upon the cortical apparatus of the Island of Reil, or to put it more accurately, greater or lesser destruction of this cortex results in more or less impairment in the function of repetition. This is strikingly borne out by the present case in which the total lack of the capacity for repetition can be correlated with the post-mortem finding of complete destruction of the Island of Reil. This parallelism has also been described by several other writers (Pick, 1898, Voisin, 1867).

In the light of this explanation, the peculiarity which our patient exhibited in his attempts at repetition, becomes understandable. As we have pointed out, in his attempts to repeat the given word, he would at times utter words which bore a definite relationship to it-e.g. "Himmel" (Heaven) for "God"; "family" for " children"; " glass" for " window," etc. Similar observations have been mentioned several times in the literature : Heilbronner (1910), Kleist (1931), Henneberg (1918), Goldstein (1906). Goldstein assumed that this behaviour is characteristic of a lesion of the Island of Reil, which makes impossible a real repetition, but which does not hinder the patient from understanding the general significance of the given word sufficiently to utter words which belong to the same "sphere."

Such a phenomenon occurs even in experience with normal individuals. We know that to grasp the general relationship or "sphere" of a heard word is an easier performance than the correct perception of the individual word. We often find ourselves in a similar situation if we are confronted with rare or foreign words. We understand in a vague way what the words relate to, i.e. in what sphere they belong, but we are not able to understand the sense of the individual words or to repeat them correctly. This happens particularly when the sphere of the word-stimulus corresponds to the situation in which we find ourselves.

The understanding of our patient was undoubtedly mainly of that type. Very often he failed to understand single words, particularly when they referred to spheres apart from the situation he was in. On the other hand, he understood relatively well sentences which belonged to the concrete situation in which he found himself. Hence strictly speaking we should modify our statement that the patient's capacity for understanding speech was relatively intact, by pointing out that although this was true in certain situations, the function of understanding speech was not really normal, but belonged to this special type of " sphere " understanding which we have just described. It is quite possible that this type of understanding is served by the right hemisphere in right-handed individuals (vide infra).

The second striking symptom which our patient exhibited, the inability to use name-words, can also be understood by the large defect in the Island of 
Reil and the adjacent gyri in the temporal and parietal lobes, since, as we know, the lesions in so-called " amnesic aphasia," with which we are dealing here, show a predilection for this part of the brain (for a detailed discussion of the localization of the lesion in amnesic aphasia (see Goldstein, K. 1927).

The localization of the lesion in the left hemisphere agrees with the fact that the patient was a right-handed individual. However, a careful review of the entire clinical picture reveals some difficulties which the above explanation does not clarify. First, how was it possible that the patient heard and understood words and even sentences relatively well, if the temporal lobe of the left side was involved to such a degree by the lesion? Not only was the so-called Wernicke relation in the first temporal convolution destroyed but also the general auditory centre of the left temporal lobe (the so-called Heschl's convolution). This defect forces us to assume that the patient's understanding of words must have been maintained by the corresponding part of the temporal lobe in the right hemisphere. However, should not the necessity of making this assumption lead us to doubt that the patient was a right-handed individual ? No. The patient's statements, and the observation of his predominant use of the right hand, made it more than probable that in essential performances he was righthanded. Furthermore, were he left-handed, it would be inconceivable that a left-sided brain lesion could produce such a severe incapacity for repetition and word-finding. Since this incapacity must be related to the left-sided lesion, it leaves no doubt as to the predominance of the left-hemisphere for the concerned speech function. Thus we have no choice but to assume that in our patient, in spite of the predominance of the left hemisphere, the integrity of the right hemisphere was able to guarantee understanding of speech, at least to a certain degree.

This would not contradict other experiences. We know from other cases that a gross defect in the capacity for understanding speech usually occurs only if-in addition to a lesion in the left temporal lobe-the pathways which connect the right temporal lobe with the left hemisphere are also damaged.

However, there is still another difficulty. Usually in cases of destruction of the Wernicke centre, and according to Goldstein, of the Insula Reili also, we find paraphasia. But our patient had no paraphasia! One might assume that his good motor speech was due to the undamaged 3rd frontal convolution, were it not for the fact that usually, even when this convolution is intact, destruction of Wernicke's area or the Insula Reili still results in paraphasia. On the other hand, were we to assume that the absence of paraphasia was due to the function of the intact speech area in the right hemisphere, we would then have to ask, why was not the right hemisphere able also to guarantee repetition and word-finding? Thus this latter assumption is difficult to maintain, and we would prefer to explain the absence of paraphasia in this case as due to the functioning of the motor speech area of the left hemisphere, perhaps in cooperation with the corresponding convolution in the right hemisphere. But does not this contradict the usual concept that correct word-speech is dependent on the function of the Insula Reili and the temporal lobe? Not necessarily. Not infrequently, even in cases with lesions in these areas, some words and 
sentences may be spoken without paraphasia-namely, such utterances which the patient has been accustomed to make without deliberation, which are delivered as mere motor automatisms. How much speech can be expressed in this way by an individual depends on his capacity for automatic motor speech. There are great individual differences. Owing to this varying capacity, a defect in other speech performances can be masked to a greater or lesser degree. Gelb and Goldstein (1924) have pointed out how we can be deceived regarding speech function in a patient with amnesic aphasia by the patient's use of his motor speech automatisms. The same mechanism can operate in a patient with paraphasia produced by a lesion of the Insula Reili or the temporal lobe. If the patient speaks little, and if his speech is largely restricted to the same wellknown words and sentences, particularly if he possesses many such - then his paraphasia might not be discovered. There is some justification for assuming that this was the case in our patient. Although he spoke fairly well, he generally did not attempt to say much and usually uttered the same phrases and words. Although a simple man, he spoke five languages, and it is probable that being a simple man, he had especially well developed his motor speech automatisms in all these languages. It must be emphasized, however, that despite his knowledge of five languages, he could not find or repeat words to command in any of them.

To summarize our hypothesis, then, we may say :-We believe that the lack of the capacity for repetition and word-finding, was caused by the lesion of the Insula Reili and adjacent convolutions in the temporal and parietal lobes of the left hemisphere-corresponding to the patient's right-handedness ; his capacity for understanding speech was probably guaranteed by the right hemisphere; his capacity for speaking without paraphasia, by the 3rd frontal convolution of the left hemisphere, perhaps in connection with the corresponding convolution of the right hemisphere.

There still remains, however, one very amazing finding in our patient which requires clarification. It is very unusual to find the capacity for repetition and word-finding disturbed selectively to as marked a degree as he exhibited. This fact demands a special explanation. We believe that the unusual extent of this disturbance in our patient took origin not only from the causes already mentioned but also from the particular change of personality which he exhibited. This change of personality has been described by Gelb and Goldstein (1924) as characteristic of patients with amnesic aphasia. According to these authors, the difficulty of finding words for naming, is only one expression of a change of the total personality. These patients have lost the "categorical" attitude and are reduced to a concrete level of behaviour. To them the normal attitude from which we name objects is entirely strange. Nominal words do not even occur to them. This is shown by the fact that our patient was able to use words in spontaneous or conversational speech which he could not utilize when asked to name an object. There was no doubt that he recognized objects. He could demonstrate how they were used, and occasionally, in explaining their relationship he would use the desired words, although he could never repeat them to demand or name the objects when they were presented to him. 
In all these phenomena the patient's behaviour was similar to that of other amnesic aphasics. Both in his routine living as well as in special tests he revealed quite clearly the concreteness of behaviour which is so characteristic of this class of patients, differing from them only in the remarkable degree to which this concreteness was developed. One of us (K. G.) who has had the opportunity of examining many cases of amnesic aphasia, has never previously seen this phenomenon so pronounced in any of them.

\section{Conclusion}

An unusual case of amnesic aphasia is described clinico-pathologically. The receptive and expressive functions of speech were relatively only mildly disturbed. There was a striking impairment, however, in the naming of objects and the capacity for repetition. Added to this, there was a striking change in personality with loss of the capacity for abstract conception.

An attempt is made to explain the clinical picture on the basis of the anatomical findings. We are aware of the complexity of this endeavour, and although not entirely satisfied with our explanations feel that what we have suggested constitutes the most acceptable hypothesis in the light of available data.

This study was done under the auspices of the Harry H. Straus Neurological Fund. study.

K. G. expresses his appreciation to the Rockefeller Foundation for special grants aiding this

The authors express their appreciation to Dr. Charles Davison for the neuropathological material used in this study.

\section{Protocols}

(1) The following protocol reveals the extent of the patient's vocabulary, his ability to make himself understood despite grammatical difficulties, and his comprehension of simple questions, particularly when they refer to his immediate situation.

The patient is co-operative, glad to see the examiner, and quite loquacious. $\mathrm{He}$ prefers a mixture of German and Yiddish, sometimes interspersed with English expressions. At times he is rather hard to understand. Paraphasia or dysarthria were not observed. His facial expression is vivid and adequate. He understands simple questions put to him in English or German. ("+" indicates a correct response)

(Your name ?) +

(Touch your ear !) +

(Where, here ?) +

(Who am I ?) (smiled, because question seems so simple). +

(Touch your eye), repeats several times " Auge, Auge." +

(Touch your ear) Idem. - +

(Touch left ear with left hand.) +

(Touch right ear with left hand.) Perplexed, touches left ear, looks at examiner, cannot succeed.

(Touch right eye with left hand) Idem. He is not able to do tests with three items, as for instance touch with left hand first left ear then left eye. Asked what he has to complain about (a mixture of German and Yiddish) "Ich kann Sie kaum es sprechen aus-Ich habe ganz viel geleren-mein Kind sing gelernen-mein Frau ist da in Behandlung-die Kinder sind hier-ich kann bleiben. Die Leute so schmutzig- 
die Finger-ich komme herein hier-sie machen so schmutzig—ich lieb nicht die Leute. Mein Sohn verheiratet, a ganz feine Mann-ich moechte that is mein Sohn-seine Frau-mein Tochter-sie ist in high school-English-ich moeg so gerne-I can speak French, too, parfaitement. I was in that position there-in 100 Street-mein wifemy children, one child was home. I got nice home-I come home-my wife-you eat something -it come 8 o'clock "Gustaf, you feel goot ?"-I feel goot. Half an hour later what happens-mein Gustaf-he's dead. I had twenty professor-manymy children all bring - he dead-cannot sprechen-Ich habe garnicht gewissen-wass -ich weisse garnicht was (what is your son doing ?). Was von mein Sohne ? was tut er ? what is he doing-ja-he was there 20 years. Er war sehr gute Mann. He has a business. (What is the doctor doing ?) He writes. (What with ?) Everything what you wish.

(Has your son such things-bottle of glue ?)-He's a printer-yes-he's got everything, he's got a nice business-he's got such things-but he has not use for dose things. Years ago-he used to make dem-but not now (pointed to book) - he does this things-he writes this things with a machine-a big machine-here, here and herehe's the boss-ya (what do other men do ?) - I know (shakes head) (printer ?)-yeshe's working over-others working for him getting \$25, \$40 a week. He does every work. He does difference work-I don't remember-I used to remember-I asked him how's business-" oh great, papa "-heute you erregnerich ?-yes it looks like rain to-day-but it's cold-the sun did not shine (does the sun shine ?)-yes, no, but I can't say-I know rain, rain it is nice warmer, warmer, colder (sky ?)-it don't look like rain. (Say the same words as I say) :

rain-Mein Herr.

rain-rain, it's raining, it's raining.

sky - I am sorry, I wish I could say every word. God, God is always there-God -I know-I understand (but the sky-it's raining) - not now-not raining no more. Gut gestern raining all night.

holidays-kosher-yo. . . yontiff, I know, I understand what is yontiff, to-morrow, Sunday, Monday, Monday it will be finished, Pesach, Easter.

(2) Manikin Test (Pintner and Paterson, 1931) : This little test, although relatively simple, involves the capacity of the patient to form a "Gestalt," i.e. to abstract a conception from a group of apparently dissociated objects. In spite of its simplicity we see that this previously highly intelligent individual had difficulties with it. Although he finally solved it more or less correctly, he had to proceed in trial and error fashion.

First he recognizes object by legs, head, and then seems to recognize readily that it is a man. Puts two legs together, using words "Fuesse, shoes." Puts two arms together, and points to his own arms. Patient then puts legs to trunk, but in wrong order-he just pushed them up without looking at the form outlined. He sees error, reverses legs, and inserts correctly. Puts left arm in right socket (it being nearest), sees mistake, corrects it. Puts other arm, then head in place. Arms put close to sides-not partially abducted. Patient quite joyous upon completion. Time : 3.15.

(3) Feature Profile Test (Pintner and Paterson, 1931) : This test is somewhat more complicated than the Manikin Test in that it involves the handling of two factors by the subject-one the grasping of the outer form of the test pieces, and the other, the grasping of lines on the test pieces which when put together properly form the eye, ear, nose, etc. The fusing of two such factors involves what we speak of as the capacity for abstract conception. As the test shows, the patient was lacking in this ability. 
Cannot say what it is by its outline form. Uses trial and error to fit forms of profile. When he fits correctly "That's right." He knows when he is right or wrong. Completes profile, then " oh, my God, a whole Kopf." First looks along outside of figure in order to fit earpieces. Must then be shown hole in centre. Starts with trial and error inside-no success - then tries to put ear together on outside. He uses the lines on the blocks-knows when he is wrong. Puts two pieces correctly, but cannot fit other two to them. He refuses to accept his own results because he knows that they are not correct. "No, sir, it's very hard, very hard." (After about $6 \frac{1}{2}$ minutes work-" shall I show you how?") Patient asked to repeat, but no success. Illustrated once more-then asked what it might be. Patient cannot say. Picks up all pieces together-“" maybe if I put it inside, I could tell you." Put ear inside, picking all four pieces together and thus putting them in place, smiles, points to his own ear and says " nose."

(4) Colour Sorting Test. This test reveals more clearly than any of the other tests the particular nature of the patient's intellectual deficiency. It will be noted that he seemed to be completely unable to grasp the idea of "colour" as an abstract conception. He was able to think only of particular shades. Thus, he could not see the categorical connection between a light green and a dark green. To him they were different colours, and under no circumstances could he be induced to realize that they belonged in the same general grouping. When he used the words " green " or " red," it became clear that he was not using the terms in their generic sense, but was referring only to the particular shade of green or red that he had under observation at the moment. Similarly, although he at times would match bright shades of different colours to a given bright shade of red, he was nevertheless totally unable to grasp the idea of " brightness" as an abstract concept, and could not pick out bright shades of different colours on demand.

The patient is asked to pick out from a heap of assorted coloured yarns all those strands which are similar in colour to a given sample.

(a) Medium green.-He picks up a medium green. "That is a heller (brighter) green. Dies heir green, das is schoener green." He picks up a very light green. "Das heller (brighter)." He does not consider it a match and puts it aside. He picks up a dark red. Looks at the green sample, and says " No." He picks up a dark blue and a dark purple. Looks at the sample. "No, das anderes (different)." Picks up a dark green, matches it with the sample, then replaces it, apparently dissatisfied with the match. Picks up a dark purple. "No, das nicht dasselbe (not the same)." Picks up a light shade of green. "Das is greener, die mehr." Replaces it in the original heap. Picks up a very similar green to the given sample-an almost perfect match. " Ja, ja, dasselbe !" Takes other greens and attempts to match them, but returns them to the heap. Feels satisfied and joyous only when he is able to match the sample with others of almost identical shade.

(b) Goes through the same procedure with a given red.

(c) An attempt is made to demonstrate to the patient what is desired of him by putting a series of different reds in one heap, and a series of different greens in another. However, the patient was apparently unable to grasp the principle involved, and refused to admit correctness of the division. He showed satisfaction only when identical or near-identical shades were matched.

(d) Given a very light blue-matches with two light shades, but is dissatisfied. "No, no, auch nicht." " Beinahe, aber nicht dasselbe (almost, but not the same)." He picks other skeins of comparable brightness-very light blue, very light purple, 
very light grey, very light grey, very light grey, very light grey, very light green, very light brown, very light blue, very light yellow. However, when asked shortly afterwards to select light shades of different colours, he is unable to do so.

(e) He is shown a light blue, a dark blue, and a dark red, and is told that the light blue and dark blue match better than the dark blue and dark red. He shook his head, saying, "No, das blue, das is ganz andere. No, das blue, everything, nicht das, nicht das."

\section{REFERENCES}

Gelb, A., and Goldstein, K. (1924). Psychol. Forsch., 6, 127.

Goldstein, K. (1906). J. Psychol. Neurol., 7, 172.

(1915). Ergebn. Neurol. Psych., 2, 441 .

(1927). Handbuch der normalen und pathologischen Physiologie, 10, 789.

(1936). J. Psychol., 2, 301.

Heilbronner, K. (1910). Handb. Neurol., $1,988$.

Henneberg, R. (1918). Neurol. Z Zbl., 37, 704.

Kleist, K. (1931). Mschr. Psychiat. Neurol., 79, 338.

Pick, A. (1898). Beitrage zur Pathologie des Zentralnervensystems, Berlin.

Pintner, R., and Paterson, D. G. (1931). A Scale of Performance Tests, p. 218. Appleton \& Co., New York. 2nd edition.

Voisin, R. (1867). Gaz. Hôp. Paris, 39, 


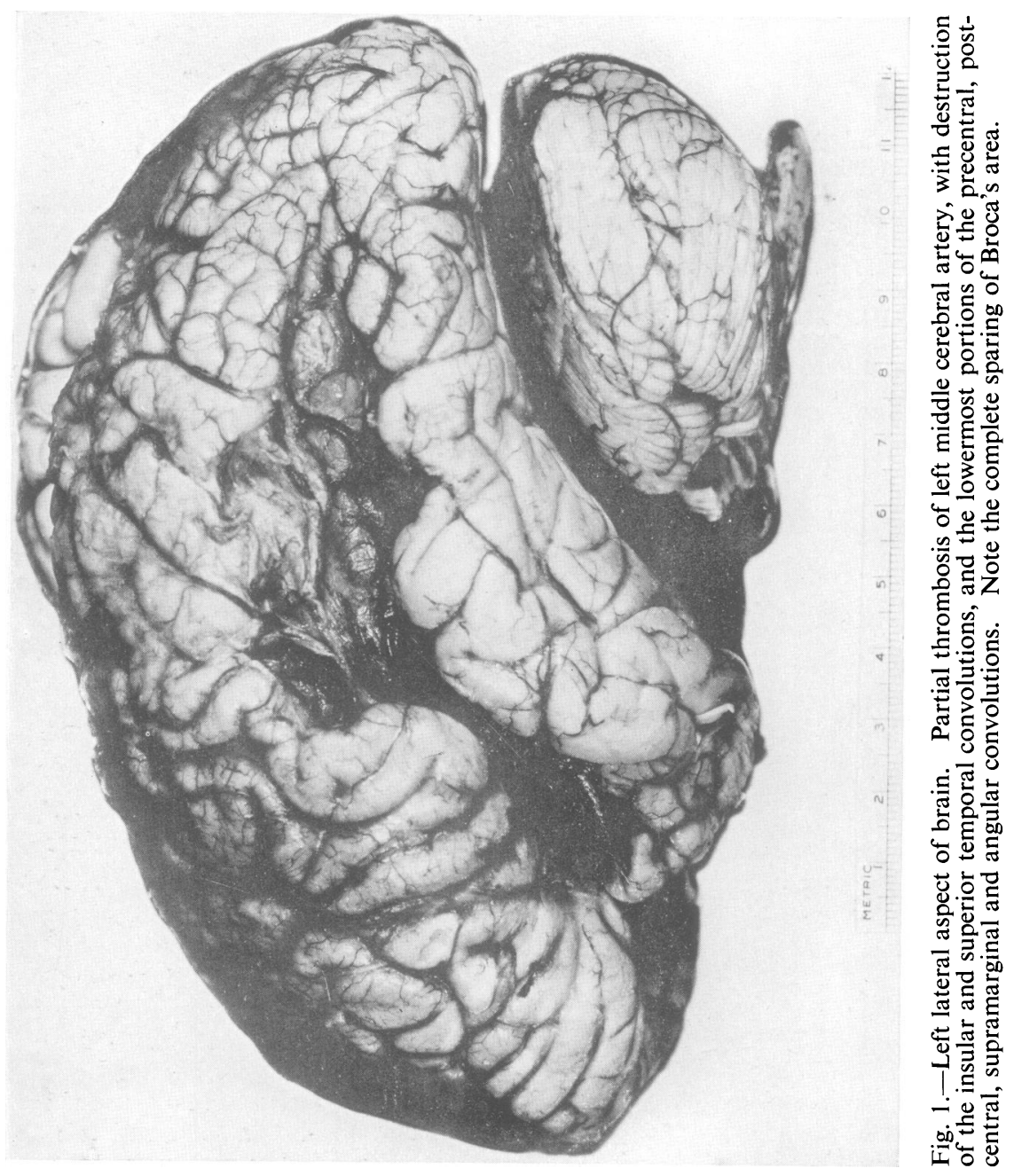




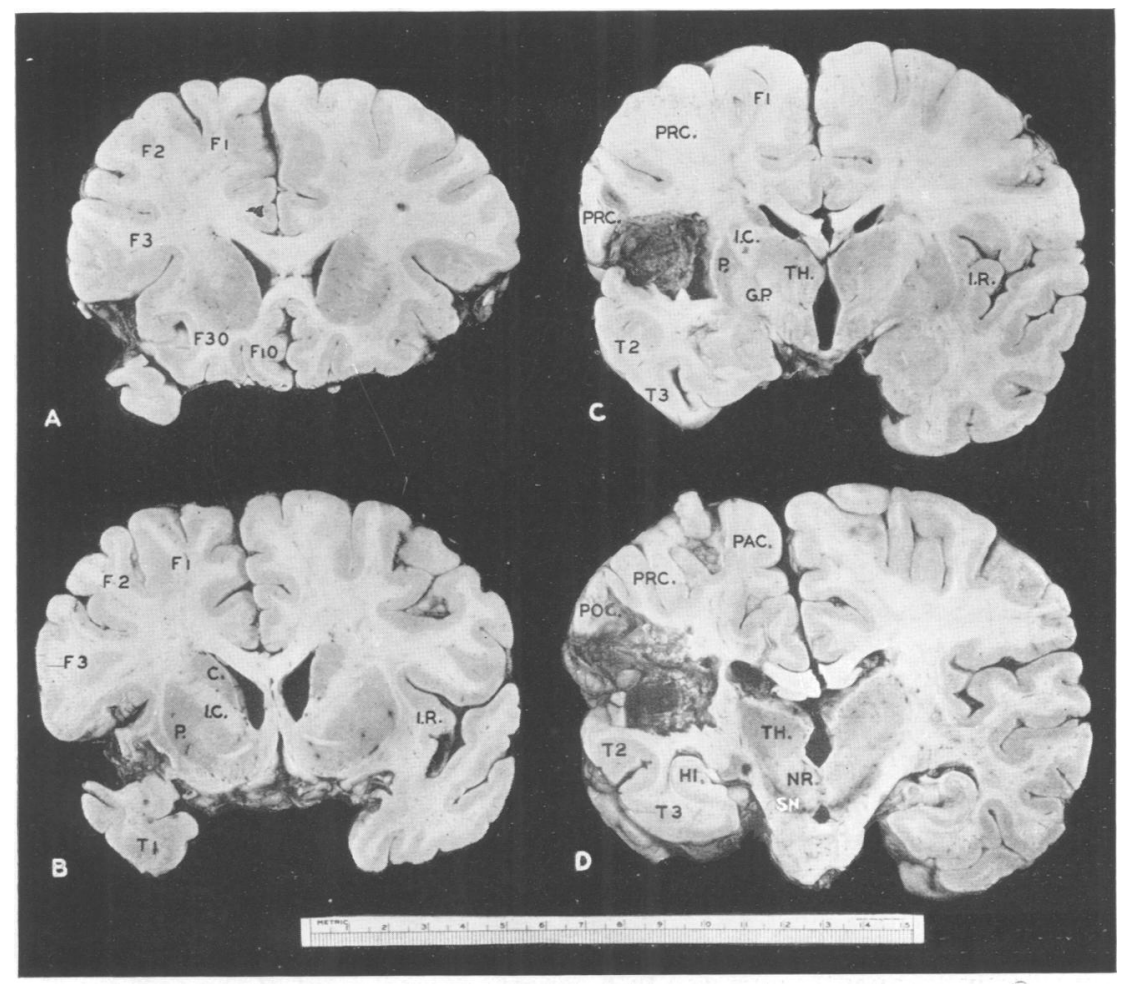

Fig. 2.-Coronal sections through the genu of the corpus callosum, the striatum, the third ventricle, and the substantia nigra. F1, F2, F3-frontal convolutions; F10, F30-orbital convolutions ; C-caudate ; I.C.-internal capsula ; I.R.-Island of Reil ; P.-putamen ; G.P.-globus pallidus ; T1, T2, T3-temporal convolutions ; TH.thalmus ; PRC.- precentral ; POC.-postcentral ; PAC.-paracentral ; HI.- hippocampus; N.R.-nucleus ruber; S.N.-substantia nigra. Note sparing of Broca's area $(\mathrm{F} 3)$.

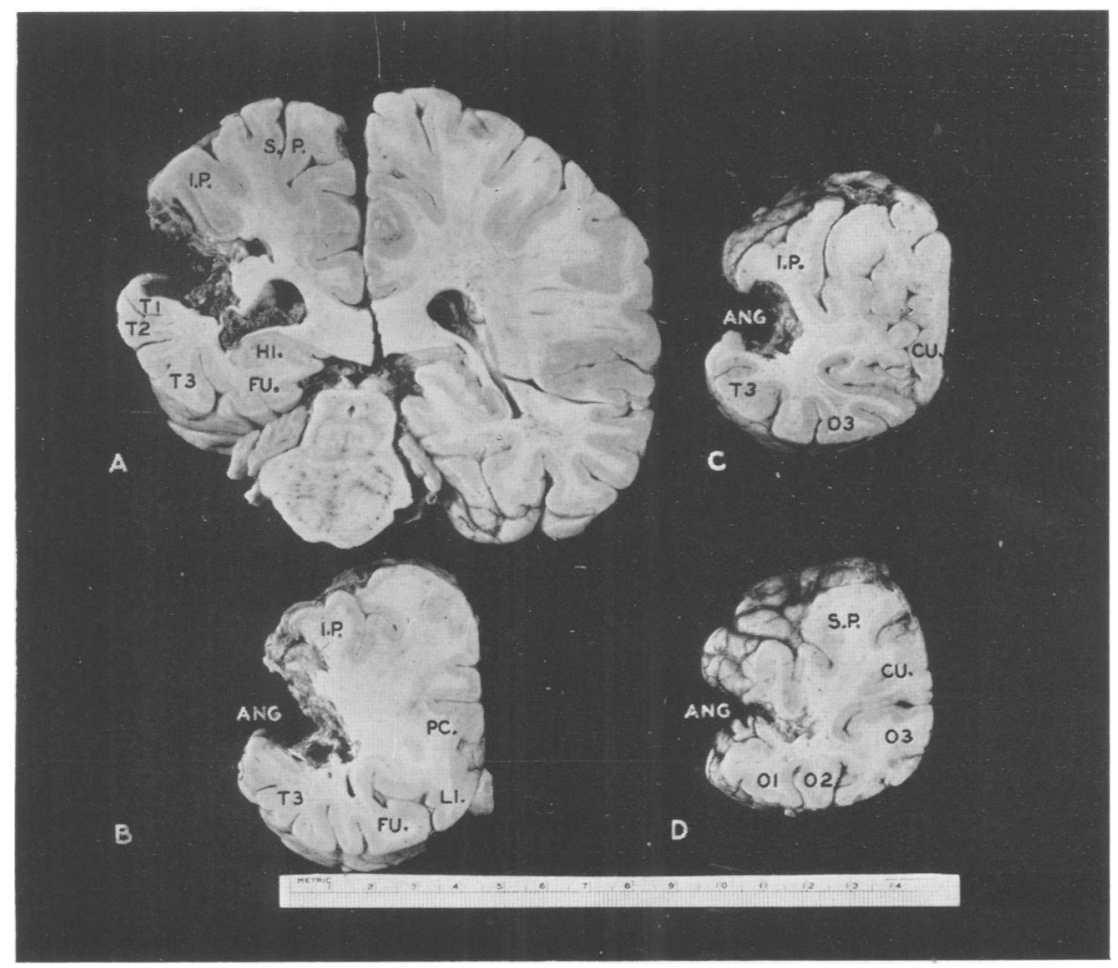

Fig. 3.-Coronal sections through the splenium of the corpus callosum, and the left occipital lobe. S.P.-sup. parietal ; I.P. inf. parietal ; H.I.-hippocampus ; FU.fusiform ; T1, T2, T3-temporal convolutions; PC.-precuneous ; L1-lingual ; CU.-cuneus ; ANG.-angular ; O1, O2, O3-occipital convolutions. 